\title{
Spontaneous diabetic myonecrosis: report of four cases from a tertiary care institute
}

\author{
Soham Mukherjee', Anuradha Aggarwal', Ashu Rastogi', Anil Bhansali', \\ Mahesh Prakash ${ }^{2}$, Kim Vaiphei $^{3}$ and Pinaki Dutta ${ }^{1}$ \\ Departments of ${ }^{1}$ Endocrinology ${ }^{2}$ Radiology ${ }^{3}$ Histopathology, Post Graduate Institute of Medical Education \\ and Research, Chandigarh 160012, India
}

Correspondence should be addressed to P Dutta

Email

pinaki_dutta@hotmail.com

\section{Summary}

Spontaneous diabetic muscle infarction (DMI) is a rare and under diagnosed complication of diabetes mellitus. Clinically it presents with acute to subacute onset swelling, pain and tenderness of muscle(s) without systemic manifestations. MRI is helpful in diagnosis, exclusion of other causes and for localization of affected muscle for biopsy in atypical cases. Muscles of the thighs are commonly affected in diabetic myonecrosis (DMN). Here we present the summary of four cases seen in the last 3 years in a tertiary care centre with simultaneous or sequential involvement of multiple groups of muscles or involvement of uncommon sites. All these patients had advanced duration of diabetes with microvascular complications and poor glycemic control. Conservative management including rest and analgesics is the treatment of choice. Short-term prognosis is good but there may be recurrence.

\section{Learning points:}

- A high index of suspicion is required for the diagnosis of DMN which can avoid inadvertent use of antibiotics.

- Acute-subacute onset severe focal muscle pain in the absence of systemic symptoms in a female patient with longstanding diabetes with microvascular complications suggests DMI.

- MRI is the most sensitive test for diagnosis.

- Muscle biopsy should be reserved for atypical cases.

- Conservative management including rest and analgesics has good outcome.

- Improvement usually occurs within 6-8 weeks, but there may be recurrence.

\section{Background}

Diabetic myonecrosis (DMN) is an unusual and under diagnosed complication of diabetes mellitus. It refers to spontaneous ischemic necrosis of muscle not related to atheroembolism or occlusion of major arteries. Angervall $\&$ Stener (1) reported the first case in 1965. Patients usually present with acute onset painful swelling of muscle(s) without any history of preceding trauma. It usually occurs in patients with long-standing diabetes with coexisting microvascular complications. Muscles of the thigh are most commonly affected. Here, we are presenting four cases of diabetic muscle infarction (DMI) that had atypical presentation in the form of simultaneous or sequential involvement of multiple groups of muscles.

\section{Case presentation}

Case summary

Case 1 A 42-year-old female patient presented with complaint of pain and swelling in the right calf for the last 2 months (Fig. 1a). There was no history of fever. She was a known case of type 2 diabetes mellitus (T2DM) for the 

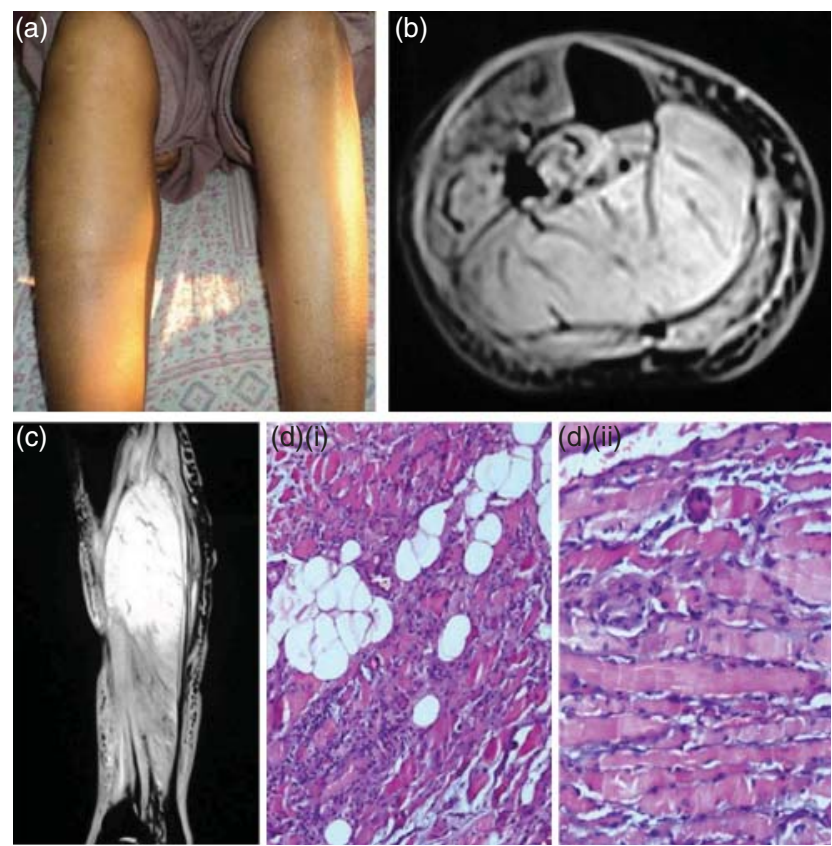

\section{Figure 1}

(a) Swelling of the right calf muscle. (b) T2W fat-saturated MRI right leg (axial view) showing diffuse hyperintensity of muscles of right calf. (c) MRISTIR sequence (coronal view) showing abnormal hyperintense signal in the calf muscles. (d) (i) Low power photomicrograph (H\&E, 140 $\times$ ) of muscle biopsy showing replacement fibrosis, fat infiltration, interfibre edema and myxoid stroma. There are variable amounts of lymphomononuclear cell infiltration forming focal aggregate. (ii) Medium power photomicrograph (H\&E, 240 $\times$ ) showing degenerated muscle fibres, interfibre edema, myxoid stroma and foreign body-type of multinucleated giant cell (at upper part of the image).

last 11 years, hypertensive for the last 1 year and hypothyroidism for the last 2 months. Patient underwent hysterectomy 3 years back. There was no history of trauma, i.m. injection, arthralgia or other systemic symptoms. On examination, right leg was swollen, tender and indurated. On evaluation, patient had all microvascular complications of diabetes. Investigation revealed hemoglobin of $7 \mathrm{~g} / \mathrm{dl}(11-15 \mathrm{mg} / \mathrm{dl})$, total leucocyte count $10200 / \mathrm{mm}^{3} \quad\left(4000-11000 / \mathrm{mm}^{3}\right)$, platelets $293 \times 10^{3} / \mathrm{mm}^{3}$, peripheral blood film suggestive of microcytic hypochromic red blood cell, urea $72 \mathrm{mg} / \mathrm{dl}$ (10-50 mg/dl), creatinine $3.2 \mathrm{mg} / \mathrm{dl}(0.5-1.2 \mathrm{mg} / \mathrm{dl})$, HbA1c 8\% (3.8-5.6\%), thyroid-stimulating hormone (TSH) $10.8 \mu \mathrm{IU} / \mathrm{ml}(0.27-4.2 \mu \mathrm{IU} / \mathrm{ml})$, thyroxine $\left(\mathrm{T}_{4}\right) 6.98 \mu \mathrm{g} / \mathrm{dl}$ $(4.8-12.7 \mu \mathrm{g} / \mathrm{dl}), \quad$ tri-iodothyronine $\quad\left(\mathrm{T}_{3}\right) \quad 0.8 \mathrm{ng} / \mathrm{ml}$ $(0.8-2.0 \mathrm{ng} / \mathrm{ml}), 24 \mathrm{~h}$ urinary protein $550 \mathrm{mg} /$ day and CK 403.5 U/1 (26-308 U/1). ANA, ANCA, RA factor, dsDNA, SSA/RO, SSB/LA, Sm, Sm/RNP, Scl70, Jo1 were negative and coagulation profile was normal. Fundus examination revealed hard exudates. Blood culture was sterile.
Deep vein thrombosis (DVT) was excluded by compression ultrasonogram (USG). USG of the right leg was suggestive of diffuse thickening and increased echogenicity of skin and subcutaneous tissue along with marked swelling and hypoechogenicity of muscles without any localized collection. MRI right leg revealed thickening and edema of the skin/subcutaneous tissue with hyperintensity in muscles of all the compartments of the right leg in short tau inversion recovery (STIR) sequence (Fig. 1b and c). Electromyography (EMG) was suggestive of inflammatory myositis. Muscle biopsy (Fig. 1d) from right gastrocnemius was performed as calf muscle involvement is relatively uncommon in DMN. Patient was diagnosed to have DMN and was treated conservatively with paracetamol and tramadol with complete recovery within 6 weeks. The dose of levothyroxine was also optimized.

Case 2 A 48-year-old female, presented with pain and swelling over her left thigh for the last 3 months and over right thigh for the last 2 weeks (Fig. 2a). There was history of similar painful swelling over right thigh 1 year back which resolved spontaneously. She did not have constitutional symptoms. There was no history of antecedent trauma or i.m. injection. She was a known case of T2DM
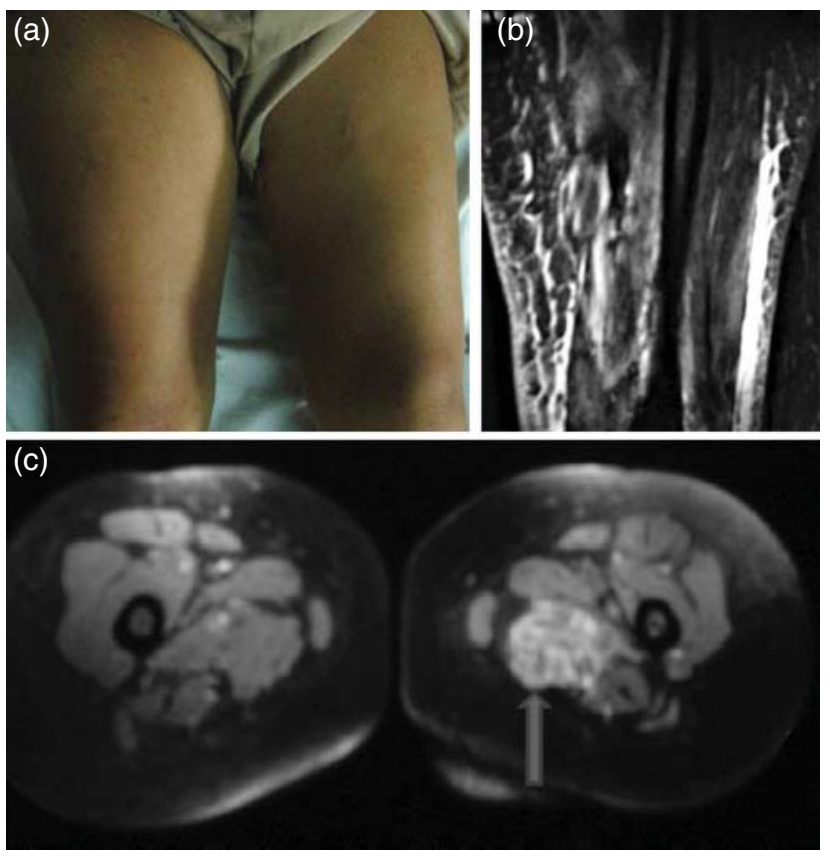

\section{Figure 2}

(a) Swelling of muscle of right thigh. (b) T2W fat sat (coronal section) shows hyperintensity of muscle with subcutaneous edema. (c) Post contrast T1W (axial section) showing enhancement with necrotic areas in the muscle. 
for the last 24 years with neuropathy, retinopathy and nephropathy, and was on continuous ambulatory peritoneal dialysis for end stage renal disease. Her right and left thigh muscles were swollen and tender. Patient had hemoglobin of $7.1 \mathrm{~g} / \mathrm{dl}$, TLC of $14900 / \mathrm{mm}^{3}$, creatinine $7.64 \mathrm{mg} / \mathrm{dl}$. Coagulation profile was normal and workup for connective tissue disorder was negative. Colour Doppler study excluded deep vein thrombosis of lower limb. On USG, anterolateral muscles of bilateral thigh and anterolateral muscles of left leg were bulky and heterogonous. MRI performed 3 months prior, revealed bulky muscle group on medial aspect of left thigh with increased intensity on T2-weighted sequence. Post contrast study revealed heterogeneous enhancement with a small noncontrast enhanced necrotic region suggestive of myonecrosis (Fig. 2b and c). Insulin therapy was optimized and i.v. iron sucrose, vitamin $\mathrm{B}_{12}$ and erythropoietin were administered for anemia. She was managed conservatively with codeine and paracetamol for 6 weeks.

Case 3 A 51-year-old female patient presented with complaint of pain and swelling in her right thigh for the last 2 weeks (Fig. 3a). She had fever and there was no history of trauma or i.m. injection. She is a known case of T2DM for the last 12 years, hypertensive for the last 1 year and hypothyroid on levothyroxine replacement for last 3 years. She had similar history with involvement of the left thigh and left calf muscle 4 and 8 months back respectively. On examination, right thigh was swollen, tender and indurated. She had neuropathy, nephropathy
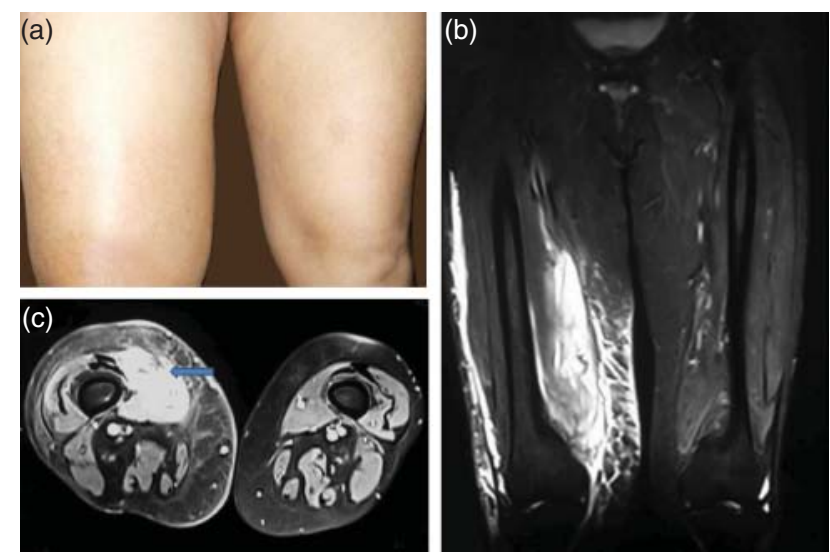

Figure 3

(a) Swelling of right thigh. (b) T2W fat-saturated MRI (coronal section) both thigh with hyperintensity in vastus medialis muscle of right thigh. (c) T1W MRI (axial section) of right thigh demonstrating post contrast enhancement of vastus medialis muscle with small areas of non-enhancement; features consistent with diabetic myonecrosis. and proliferative retinopathy. Investigation revealed hemoglobin $10.9 \mathrm{~g} / \mathrm{dl}$, TLC $11200 / \mathrm{mm}^{3}$, platelets $245 \times 103 / \mathrm{mm}^{3}$, peripheral blood film suggestive of microcytic hypochromic red blood cell, urea $28 \mathrm{mg} / \mathrm{dl}$, creatinine $1.0 \mathrm{mg} / \mathrm{dl}, \mathrm{HbA} 1 \mathrm{c} 11.2 \%$, TSH $9.59 \mu \mathrm{IU} / \mathrm{ml}, \mathrm{T}_{4}$ $7.98 \mu \mathrm{g} / \mathrm{dl}, \mathrm{T}_{3} 1.02 \mathrm{ng} / \mathrm{ml}$ and CK $1281 \mathrm{U} / \mathrm{l}$. Rheumatologic work-up was negative and coagulation profile was normal. $24 \mathrm{~h}$ urinary protein excretion was $642 \mathrm{mg} /$ day. DVT was excluded by compression USG. USG right thigh showed generalized hypoechogenecity in the quadriceps muscles without any evidence of collection. MRI bilateral thigh revealed diffuse ill-defined intramuscular (right more than left) heterogeneous, intermediate to increased T2WI-IR (inversion recovery) and reduced T1 intensity, and significant contrast enhancement to suggest inflammatory etiology (Fig. 3b and c). There was involvement of the entire length of muscles of anteromedial compartment of the right thigh with dispersed areas of low intensity post contrast - suggestive of necrosis along with overlying subcutaneous fat edema - infiltration, while there was much limited involvement of muscles of medial compartment of the left thigh. The MRI features were consistent with the diagnosis of DMN. The patient was managed conservatively and doses of insulin and levothyroxine were optimized.

Case 4 A 43-year-old female patient, known case of T2DM and hypertension for last 9 years, presented with complaint of pain and swelling over right thigh for the last 2 months. She had similar history of pain and swelling of left thigh 1 year back which got relieved spontaneously. She had diabetic kidney disease and was dialysis dependent. There was diffuse swelling over lateral aspect of her right thigh which was tender. Investigation revealed hemoglobin of $6.2 \mathrm{~g} / \mathrm{dl}$, normal total leucocyte count, urea $102 \mathrm{mg} / \mathrm{dl}$, creatinine $5.7 \mathrm{mg} / \mathrm{dl}$, HbA1c 7.1\% and CK 113.5 U/l. Autoimmune markers including ANA, dsDNA, SSA/RO, SSB/LA, Sm, Sm/RNP, Scl70, Jo1 were negative. DVT was excluded by compression USG. USG right thigh revealed generalized hypoechogenecity in the quadriceps muscles without any evidence of collection. The MRI thigh was suggestive of myonecrosis. In view of atypical presentation excision biopsy from muscle of right thigh was done which was suggestive of large areas of scarring with caught up fibrosis in various stages of compensation suggestive of post-necrotic scarring. Patient improved with conservative management within 8 weeks. Four months later, patient developed similar episode involving left calf muscle which also improved with conservative approach. 


\section{Discussion}

In this small series we report on four patients of DMN (Table 1). The first patient had isolated calf muscle involvement while the third and fourth patients developed DMI in various muscles groups (thigh with or without calf muscle) at different time frames. The second patient had simultaneous involvement of multiple groups of muscles. All these patients had long-standing diabetes with diabetic microvascular complications as a common factor. The last case has been previously reported by us (2).

Two of our patients had associated hypothyroidism. One of them was already on treatment with normal $\mathrm{T}_{4}$ and the other one was having sub-clinical hypothyroidism for the first time. In both of them the treatment was optimized. The association of hypothyroidism with myalgia and elevated CK levels is well documented in the literature; however, it is usually seen in those patients with long-standing untreated hypothyroidism. Further, acute-onset symptoms, focal involvement of specific group of muscles and features of SIRS are not the usual presenting manifestation of hypothyroidism.

Statin induced myopathy is not plausible in any of our patient as all of them were statin naïve at the time of presentation.

DMN or DMI is a rare complication of diabetes mellitus. It is more common in patients with T1DM and women with T2DM (3). It occurs in patients with longstanding diabetes ( $>15$ years) along with microvascular complications including retinopathy (71\%), nephropathy (57\%) and/or neuropathy (55\%). Patients usually present at a mean age of 40 years with acute or subacute onset pain, tenderness and edema of muscles (3). Muscles of thigh and hip are more commonly involved, with the most common muscle being quadriceps (60-65\%), followed by hip adductors (13\%), hamstrings (8\%) and hip flexors (2\%). Further, calf muscles may also be affected in DMN (4). In our series, calf muscles were involved in three patients. In the literature, there are only few case reports of unusual sites of muscle involvement in DMN. Martinez et al. (5) reported a case of DMN where proximal muscles of both upper and lower limbs were involved. Similarly Mukhopadhyay et al. (6) reported a patient with $\mathrm{DMN}$, in whom proximal muscles of both upper limbs were involved. DMN or DMI is a rare complication of diabetes mellitus. It is more common in patients with T1DM and women with T2DM (3). It occurs in patients with long-standing diabetes ( $>15$ years) along with microvascular complications including retinopathy (71\%), nephropathy (57\%) and/or neuropathy (55\%). Patients usually present at a mean age of 40 years with acute or subacute onset pain, tenderness and edema of muscles (3). Muscles of thigh and hip are more commonly involved, with the most common muscle being quadriceps (60-65\%), followed by hip adductors (13\%), hamstrings $(8 \%)$ and hip flexors (2\%). Further, calf muscles may also be affected in DMN (4). In our series, calf muscles were involved in three patients. In the literature, there are only few case reports of unusual sites of muscle involvement in DMN. Martinez et al. (5) reported a case of DMN where proximal muscles of both upper and

Table 1 Summary of four cases with DMN.

\begin{tabular}{|c|c|c|c|c|}
\hline Parameters & Patient 1 & Patient 2 & Patient 3 & Patient 4 \\
\hline Gender & $\mathrm{F}$ & $\mathrm{F}$ & $\mathrm{F}$ & $\mathrm{F}$ \\
\hline Age (years) & 42 & 48 & 51 & 43 \\
\hline Diabetes & Type 2 & Type 2 & Type 2 & Type 2 \\
\hline Duration (years) & 11 & 24 & 12 & 9 \\
\hline Nephropathy & + & + & + & + \\
\hline Retinopathy & + & + & + & + \\
\hline Nephropathy & + & + & + & + \\
\hline Comorbities & $\begin{array}{l}\text { HTN } \\
\text { Hypothyroidism }\end{array}$ & HTN & $\begin{array}{l}\text { HTN } \\
\text { Hypothyroidism }\end{array}$ & HTN \\
\hline Muscle group & Right calf & $\begin{array}{l}\text { Anterolateral group of } \\
\text { muscle of both thigh } \\
\text { Medial group of muscle } \\
\text { of left thigh } \\
\text { Anterolateral group of } \\
\text { muscle of left leg }\end{array}$ & $\begin{array}{l}\text { Anteromedial group of } \\
\text { muscle in right thigh } \\
\text { Medial group of muscle } \\
\text { in left thigh }\end{array}$ & $\begin{array}{l}\text { Anterolateral group of } \\
\text { muscle of right thigh }\end{array}$ \\
\hline MRI & s/o myonecrosis & s/o myonecrosis & s/o myonecrosis & s/o myonecrosis \\
\hline Treatment for DMN & $\begin{array}{l}\text { Paracetamol and } \\
\text { tramadol (6 weeks) }\end{array}$ & $\begin{array}{l}\text { Codeine and } \\
\text { paracetamol ( } 6 \text { weeks) }\end{array}$ & $\begin{array}{l}\text { Paracetamol and } \\
\text { tramadol ( } 6 \text { weeks) }\end{array}$ & $\begin{array}{l}\text { Paracetamol and } \\
\text { tramadol ( } 6 \text { weeks) }\end{array}$ \\
\hline Outcome & Improved & Improved & Improved & Improved \\
\hline
\end{tabular}


lower limbs were involved. Similarly Mukhopadhyay et al. (6) reported a patient with DMN, in whom proximal muscles of both upper limbs were involved.

Patients with DMI commonly present with tender and swollen muscles with elevated inflammatory markers and normal or mildly elevated creatine phosphokinase (CPK) (7). DVT, hematoma, abscess, pyomyositis, fasciitis, myositis and malignancy should be considered as differential diagnosis. Inclusion body myositis, an autoimmune disorder is another close differential diagnosis. Sub acutechronic onset, gradually progressive proximal muscle weakness (with distal muscle involvement later-on), typical histological findings - rimmed vacuoles, mononuclear cell infiltrates and filamentous inclusions, generalized myopathic pattern on EMG and lack of contrast enhancement of the involved muscle is highly suggestive of IBM. Our first patient presented with diffuse globular swelling of right calf with involvement of muscles of each compartment, thereby clinically mimicking DVT of the right leg. MRI usually clinches the diagnosis and where diagnostic uncertainty exists, muscle biopsy may be considered (7). Typical MRI finding include diffuse enlargement of involved muscles, partial loss of normal fatty intermuscular septae and small, focal, rim-enhancing fluid collections. T1-weighted sequence demonstrates loss of normal intramuscular septae (8). T2-weighted images are the best tool to visualize subcutaneous edema, subfascial fluid, and specific muscle involvement (9). T2-weighted MRI sequence has a sensitivity of about $90 \%$ for the diagnosis of active muscle disease but it has a specificity of only 43\% for muscle infarction (10). Open muscle biopsy remains the gold standard, with a specificity of $\sim 95 \%$ (10). However, it is associated with poor wound healing and a higher risk of infection, seroma and haematoma. Therefore, currently it is recommended to make the diagnosis of DMN on the basis of clinical presentation and radiological findings; open muscle biopsy should be limited to atypical cases only (7). Histological findings of DMN include: edematous connective tissue and fascia, small pockets of edematous fluid deep to fascia with no evidence of pus, pale and woody muscles. Abundant lymphocytic infiltration and patchy atrophic fibers with surrounding fibrosis may also be seen (8). Two of our patients underwent biopsy as they had relatively atypical presentation. Some authors recommended MRI guided muscle biopsy which may be target specific and reduce the risk of complications (8). Contributing factors for poor wound healing in this subset of patients are decreased cellular and growth factor response, alterations in macrophage function, poor quality of collagen and granulation tissue, abnormal keratinocyte and fibroblast migration and proliferation, and disturbance in balance between the accumulation of extracellular matrix components and their remodeling by mixed metalloproteinases (9) (11).

Pathogenesis of DMN is not well understood. Atherosclerosis, diabetic microangiopathy, ischemia-reperfusion injury and vasculitis with thrombosis have been implicated in the pathogenesis of DMI (9) (11). Others have proposed alterations in coagulation cascade (increased factor VII and plasminogen activator inhibitor activity) and occlusion of small vessels due to atheroembolism as the potential mechanisms (12). Few patients of DMI have also been found to have antiphospholipid antibody (13). However, atherosclerotic vascular obliteration appears to be the most widely accepted hypothesis. Trimodal treatment strategies have been described: supportive (bed rest, non-nephrotoxic analgesics), medical (anti-platelet agents, steroids) and rarely surgical exploration. In a study, the mean duration of recovery for the three strategies was 5.5, 8.1 and 13 weeks respectively (12). Hence, supportive management has the best outcome. However, recurrence rate are reported to be high (40\%) with a 2 year mortality rate of $10 \%(12)$.

\section{Conclusion}

Myonecrosis is a rare complication of diabetes and require high index of suspicion for the diagnosis which avoids indiscriminate use of antibiotics. It should be considered in patients of diabetes mellitus with long duration of the disease with accompanying microvascular complications who present with acute-subacute onset severe focal muscle pain in the absence of systemic symptoms. MRI is the most sensitive test for diagnosis. Muscle biopsy should be reserved for atypical cases only. Tight glycemic control, bed rest and analgesia are the cornerstone of treatment. Most of our patients had atypical presentation with synchronous/ metachronous involvement of multiple groups of muscles.

\section{Declaration of interest}

The authors declare that there is no conflict of interest that could be perceived as prejudicing the impartiality of the research reported.

\section{Funding}

This research did not receive any specific grant from any funding agency in the public, commercial or not-for-profit sector.

\section{Patient consent}

Written, informed consent was obtained from all the patients for publication of this report. 
Endocrinology,

Diabetes \& Metabolism

CASE REPORT
S Mukherjee and others

Spontaneous diabetic

myonecrosis
ID: 15-0003; May 2015

DOI: 10.1530/EDM-15-0003
Author contribution statement

All authors have contributed equally.

\section{References}

1 Angervall L \& Stener B 1965 Tumoriform focal muscular degeneration in two diabetic patients. Diabetologia $139-42$. (doi:10.1007/BF01338714)

2 Rastogi A, Bhadada SK, Saikia UN \& Bhansali A 2011 Recurrent diabetic myonecrosis: a rare complication of a common disease. Indian Journal of Medical Research 65 311-315. (doi:10.4103/0019-5359.107392)

3 Trujillo-Santos AJ 2003 Diabetic muscle infarction: an underdiagnosed complication of long-standing diabetes. Diabetes Care 26 211-215. (doi:10.2337/diacare.26.1.211)

4 Bunch TJ, Birskovich LM \& Eiken PW 2002 Diabetic myonecrosis in a previously healthy woman and review of a 25-year Mayo Clinic experience. Endocrine Practice 8 343-346. (doi:10.4158/EP.8.5.343)

5 Martinez JH, Torres O, Mangual Garcia MM, Palermo C, de Lourdes Miranda M, Gonzalez E, Espinoza IC, Laboy I, Miranda M, Dávila Ket al. 2013 Diabetic myonecrosis: an atypical presentation. Case Reports in Endocrinology 2013 190962. (doi:10.1155/2013/190962)

6 Mukhopadhyay P, Barai R, Philips CA, Ghosh J \& Saha S 2011 An unusual case of myonecrosis. Case Reports in Endocrinology 2011 624020. (doi:10.1155/2011/624020)
7 Bhasin R \& Ghobrial I 2013 Diabetic myonecrosis: a diagnostic challenge in patients with longstanding diabetes. Journal of Community Hospital Internal Medicine Perspectives 3 1-4. (doi:10.3402/jchimp.v3i1. 20494)

8 Jelinek JS, Murphey MD, Aboulafia AJ, Dussault RG, Kaplan PA \& Snearly WN 1999 Muscle infarction in patients with diabetes mellitus MR imaging findings. Radiology 211 241-247. (doi:10.1148/radiology. 211.1.r99ap44241)

9 Nagdev A, Murphy M \& Sisson C 2008 Bedside ultrasound for the detection of diabetic myonecrosis. American Journal of Emergency Medicine 26 969.e3-969.e4. (doi:10.1016/ajem2008 02.017)

10 Morcuende JA, Dobbs MB, Crawford H \& Buckwalter JA 2000 Diabetic muscle infarction. Iowa Orthopaedic Journal 20 65-74.

11 Brem H \& Tomic-Canic M 2007 Cellular and molecular basis of wound healing in diabetes. Journal of Clinical Investigation 117 1219-1222. (doi:10.1172/JCI32169)

12 Kapur S \& McKendry RJ 2005 Treatment and outcomes of diabetic muscle infarction. Journal of Clinical Rheumatology: Practical Reports on Rheumatic \& Musculoskeletal Diseases 11 8-12. (doi:10.1097/01.rhu. 0000152142.33358.f1)

13 Palmer GW \& Greco TP 2001 Diabetic thigh muscle infarction in association with antiphospholipid antibodies. Seminars in Arthritis and Rheumatism 30 272-280. (doi:10.1053/sarh. 2001.19961)

Received in final form 2 March 2015

Accepted 22 April 2015 